\title{
ESTUDIO COMPARATIVO DE LOS CRÉDITOS OTORGADOS A LA ACTIVIDAD PRODUCTIVA POR LA BANCA PÚBLICA Y PRIVADA EN LA TRONCAL, ECUADOR
}

\section{COMPARATIVE STUDY OF CREDITS GRANTED TO PRODUCTIVE ACTIVITY BY PUBLIC AND PRIVATE BANKS IN LA TRONCAL, ECUADOR}

Jessica Mariela Córdova Uzhca ${ }^{1}$

https://orcid.org/0000-0002-3550-858X jcordovau@ulvr.edu.ec.

\begin{abstract}
RESUMEN
La relevancia del tema de estudio se deriva de la finalidad de desarrollar mejores medidas de crecimiento económico en el Cantón La Troncal, siendo determinantes los créditos productivos otorgados en las instituciones financieras públicas y privadas del país para su alcance, con los cuales, se busca conseguir lo expuesto en el Objetivo de Desarrollo Sostenible 8 "Fomentar el crecimiento económico sostenido, inclusivo y sostenible, el empleo pleno y productivo, y el trabajo decente para todos". Para esto, el Estado ecuatoriano emplea diferentes normas o leyes como la Ley de Fomento Productivo, que se orienta en favorecer las medidas de créditos y pagos de los empresarios para atraer a nuevas inversiones, se puede indicar que esta forma de actuación o intervención tiene un profundo enfoque teórico mercantilista por la necesidad de intervención del gobierno para generar riquezas en los factores de producción. Por su parte, el estudio presenta una justificación teórica debido a la importancia que tienen las facilidades de crédito para incentivar los proyectos de las diferentes naciones y como se ocasionaría un progreso lento en la economía si estas prestaciones imponen mecanismos inapropiados. Además, se presenta una justificación metodológica fundamentada en una investigación descriptiva de enfoque cuantitativo ya que se aplicó una encuesta a 68 emprendedores y se realizó una entrevista a 2 instituciones financieras privadas y 2 públicas, las mismas que servirán de base para el desarrollo de futuros estudios que muestren un tema y fenómeno de investigación similar al expuesto en la presente investigación..
\end{abstract}

Palabras clave: Instituciones financieras públicas y privadas, estado ecuatoriano, créditos productivos, crecimiento económico.

\section{ABSTRACT}

\footnotetext{
${ }^{1}$ Universidad Laica Vicente Rocafuerte de Guayaquil. Facultad de Ciencias Sociales y Derech. Carrera de economía
} 
The relevance of the subject of study is derived from the purpose of developing better measures of economic growth in the Canton La Troncal, the productive credits granted in the public and private financial institutions of the country being decisive for its scope, with which it seeks to achieve what is stated in Sustainable Development Goal 8 "Promote sustained, inclusive and sustainable economic growth, full and productive employment, and decent work for all." For this, the Ecuadorian State uses different norms or laws such as the Productive Development Law, which is oriented towards favoring the credit and payment measures of entrepreneurs to attract new investments, it can be indicated that this form of action or intervention has a deep mercantile theoretical approach due to the need for government intervention to generate wealth in the factors of production. For its part, the study presents a theoretical justification due to the importance of credit facilities to encourage the projects of different nations and how slow progress in the economy would be caused if these benefits impose inappropriate mechanisms. In addition, a methodological justification based on descriptive research with a quantitative approach is presented, since a survey was applied to 68 entrepreneurs and an interview was carried out with 2 private and 2 public financial institutions, which will serve as the basis for the development of future studies. that show a topic and research phenomenon similar to the one exposed in this research.

Keywords: Public and private financial institutions, Ecuadorian state, productive credits, economic growth.

\section{INTRODUCCIÓN}

La crisis económica originada a nivel del globo que tuvo como suceso en el 2007-2008 en los mercados financieros de New York y Londres que son considerados las capitales del capital (recurso monetario), desencadenándose y expandiéndose en todas las naciones, sufriendo una recesión en los países de grandes economías y los emergentes, esto debido a las variaciones de la moneda, en el cual, Ecuador como país dolarizado no podía efectuar políticas monetarias como contramedida a los sucesos (Aguilera, 2015).

El nivel de pobreza dentro del 2008 era de 35,1\% que se incrementó a 36\% en el 2009 , estas cifras bajarían con el pasar del tiempo llegando al 2014 a representar 22,5\% así lo detalla la Encuesta Nacional de Empleo, Desempleo y Subempleo (ENEMDU , 2020). Posteriormente, para el año 2015 también se observa una afectación por la baja en el precio del crudo, en este periodo el incremento del PIB fue solo de $0,1 \%$ mientras que para el 2016 equivalía a $-1,6 \%$, esto debido a las afectaciones del terremoto que generaron estragos en las finanzas del país así lo menciona (Guilcaso, Ronquillo, \& Montaluisa, 2018), todos estos acontecimientos provocaron que los ciudadanos se vieran afectados, su grado de riqueza, aumentado la pobreza en $23,7 \%$.

En la actualidad, debido a la afectación de la pandemia el nivel de pobreza representa el $32,4 \%$ de acuerdo con la (ENEMDU , 2020), entre otros indicadores que se observan en el territorio local, se puede mencionar que el nivel de analfabetis mo es del $7 \%$ lo indica el (Banco Mundial, 2019), mientras que la tasa de delincuencia presenta una variación del $36,8 \%$ del 2019 al 2020 según él (INEC, 2021).

A nivel de La Troncal esta afectación es evidente al solo poseer un 38\% de PEA donde prevalece la Agricultura, Caza y Pesca con el 32,6\%, mientras que el nivel de analfabetismo es 
bajo con un 7,66\% así lo afirma el Plan de Desarrollo Y Ordenamiento Territorial Del Cantón La Troncal (PDOT La Troncal, 2014), además, el nivel de delincuencia expresado en la localidad fue de 5,4\% según datos registrados en él (INEC, 2011). Todos estos aspectos demuestran una económica vulnerable, que necesita de la generación de empleos para eliminar la pobreza y que no existan personas que recuran a delitos para obtener una fuente de obtención de dinero, por esto el emprendimiento se observa como un mecanismo para mejorar la economía de los ciudadanos.

La creación de emprendimientos y nuevos negocios son los mecanismos utilizados por las personas para conseguir una fuente de ingresos, para lo cual, diseñan una planificación de los recursos que necesitaran para comenzar la actividad, en este ámbito, incurren en créditos de instituciones financieras para poder efectuar su idea comercial, no obstante, no todos poseen la misma facilidad de acceder a dichos prestamos como consecuencia de las medidas planteadas por las entidades como bancos o cooperativas. López y Farías (2018) afirma que:

Las pequeñas y medianas empresas han atravesado distintos desafíos entre ellos la dificultad para acceder al crédito por que muchas de estas empresas empiezan como modelos de emprendimientos informales y no cuentan con uno de los requisitos más fundamentales que es ser legalmente constituidas. (pág. 2)

Por ende, el acceso a los créditos ha presentado de forma precedente una dificultad para los empresarios, quienes, tienen que acatar las medidas de las instituciones financieras para obtener el recurso monetario que le permita poner en marcha su negocio.

\section{Historia del sistema financiero}

Los sistemas financieros, no obstante, de observarse movimientos $u$ acciones de este tipo desde siglos precedentes a los años 1600, la conformación compleja y totalmente estructurada se remonta al periodo de 1609 con la aparición del Banco de la ciudad de Barcelona y Ámsterdam, sin embargo, los bancos de emisión se contemplan en el siglo XVIII con una mayor generalización en el siglo XIX, así lo considera (Posso, 2016).

A nivel de Latino América se desarrollaron de forma creciente los bancos entre el periodo de 1850 y 1873, conformando cerca de 90 instituciones entre los países de Argentina, Brasil, Chile, Cuba y Perú; no obstante, muchas quebraron por la crisis suscitada en la región para 1870, teniendo una recuperación para 1880. En este periodo, se autoriza a la banca privada la emisión de billetes de banco respaldados por reservas metálicas de plata, suscitando este aspecto en la nación mexicana (Marichal \& Gambi, 2017).

Por su parte, en el Ecuador los inicios del sistema financiero se dieron para 1831 en donde se estableció la institución Casa de la Mondeo en Quito, la cual, se fortalecería con la introducción de negociación para el desarrollo de una entidad bancaria, su establecimiento no fue muy estructurado, para 1839 se decreta la autorización para que el Ejecutivo gestione los fondos que permitan la creación de una institución bancaria en el ámbito local. Todo esto da origen para 1860 al Banco Particular y Banco Luzurraga, así lo menciona (Reinoso, 2018).

Durante los años 90 la economía ecuatoriana y el sistema financiero paso por muchas adversidades, siendo la más resaltable la sucedida en el gobierno de Mahuad con la aprobación de la Ley de Reordenamiento en Materia Económica en el Área TributariaFinanciera; que imponía el cobro del $1 \%$ a la circulación de capitales (ICC), lo que generó una disminución en los volúmenes de captaciones, señala (Reinoso, 2018). 
Esta situación provocó la caída en los depósitos para las instituciones financieras como derivación de que los agentes económicos a nivel nacional trataban de invadir el pago del ICC, por lo que sucedió la quiebra de los bancos, tomándose como decisión el congelamiento de los depósitos y el denominado "Feriado Bancario", así lo considera (Reinoso, 2018).

Para el 2001 se decreta la Ley General de Instituciones del Sistema Financiero que sustituye a la Ley de instituciones financieras de 1994, regulándose de forma más estricta creación, organización, actividades, funcionamiento y extinción de las instituciones del sistema financiero, como plantea (Reinoso, 2018).

Además, para asegurar la correcta administración monetaria se establece el Código Orgánico Monetario y Financiero para el 2014, como expresa (Reinoso, 2018). Lo que permite denotar una evolución en las leyes locales para abarcar diferentes aspectos financieros como los créditos y control monetario para que se garantice el correcto desarrollo del sector.

\section{Instituciones financieras}

Las instituciones financieras son las entidades del sector público o privado que brindan crédito a las personas naturales o empresas para solventar una necesidad comercial o productiva. De acuerdo con Tello, Hernani y Limaco (2017) las entidades financieras deben tener capacidad transaccional para la ejecución de sus servicios, por lo cual, menciona que debe contener las siguientes características:

- Buena relación con clientes para adaptar el producto a las necesidades que presentan y reducir el acceso al servicio que se ofrece.

- Poder de negociación para influenciar en los términos del contrato a cerrar con el cliente y mantener abierta una posibilidad de renegociación.

Dentro del tipo de instituciones financieras que se encuentran en un mercado están los bancos y las cooperativas de ahorro y crédito, lo que comprende cada una se explica a continuación.

\section{a) Bancos}

De acuerdo con Ortega (2015) los bancos "se caracterizan principalmente por ser intermediarios en el mercado financiero, en el cual actúan de manera habitual, captando recursos del público para obtener fondos a través de depósitos o cualquier otra forma de captación", (pág. 3). Con la finalidad es disponer de recursos de forma total o parcial para gestionar operaciones de crédito e inversión.

Es decir, son entidades que se financian con el dinero de los depositantes y la utilizan en operación de inversión que den créditos a la institución y a sus asociados, brindando servicios no solo de otorgamiento de crédito, también de inversión, cuentas de ahorro y cuentas corrientes.

La aparición de bancos privados se traslada desde 2.000 años antes de Cristo, lo que permite aseverar que la existencia de estos mecanismos presenta 4.000 años de datos, siendo los siglos XV, XVI y XVII en el que se potenciaron como consecuencia del sistema mercantilista de las naciones, así lo considera (Collaguazo, 2017).

\section{b) Cooperativas de ahorro y crédito}


Se entiende como una sociedad cooperativista que sustenta sus operaciones por las aportaciones que brindan sus socios, a los cuales, se les ofrece servicios de ahorro y préstamos, teniendo como característica principal ser instituciones sin fines de lucro. Desde el punto de vista de Cunalata y Quijije (2016):

Las cooperativas de ahorro y créditos son denominadas entidades financieras que ejecutan diversas actividades propias de crédito, cuyo objetivo es el de satisfacer las necesidades financieras de sus clientes o terceros que deseen involucrarse, estas cooperativas pueden ser rurales y locales, eso no cambia que tengan acceso a los fondos externos, que pertenecen a los administradores. (pág. 20)

\section{Instituciones financieras en Ecuador}

A lo largo de la historia la banca ecuatoriana ha pasado por diversas situaciones, pasando por los años 70 en una reestructuración producto de la intervención en recursos tecnologías y viéndose posteriormente afectada por la crisis del sector, en este sentido, Carillo, Mancero y Mancero (2019) menciona que "Tras la crisis bancaria ecuatoriana de 1999, disminuyó en casi un 50 \% la cantidad de instituciones, debido a las quiebras, fusiones y a la reducción de las intermediarias financieras, las sociedades financieras y las compañías financieras", (pág. 5).

A nivel de Ecuador estas entidades se rigen bajo el marco legal de la Ley de Instituciones del Sistema Financiero, como lo expresa Lovato (2015) en el siguiente párrafo:

Regula la creación, organización, actividades, funcionamiento y extinción de la las instituciones del sistema financiero privado, así como la organización y funciones de la Superintendencia de Bancos. Las instituciones financieras públicas, las compañías de seguros y de reaseguros se rigen por sus propias leyes; en lo relativo a su creación, actividades, funcionamiento y organización. (pág. 14)

Por lo cual, las organizaciones vinculadas a esta actividad deben regular su proceso conforme a los que se expresa en los lineamientos de esta norma para no incurrir en sanciones o infracciones por prácticas inadecuadas en la realización de sus funciones a nivel local. La conformación del sistema financiero ecuatoriano se puede visualizar en la siguiente tabla:

\section{Tabla 1}

Instituciones financieras locales

Instituciones Financieras Públicas

Instituciones Financieras Privadas

- Banco Central del Ecuador.

- Banco del Estado.

- BanEcuador

- Banco ecuatoriano de la Vivienda.

- Corporación Financiera Nacional.

- Bancos.

- Sociedades Financieras.

- Asociaciones y Mutualistas de Ahorro y Crédito para la vivienda.

- Cooperativas de Ahorro y Crédito.

- Almacenes generales de Depósito.

- Compañías de Arrendamiento Mercantil.

- Casas de Cambio.

Nota: Esta tabla fue adaptado de Lovato (2015) y muestras las instituciones financieras públicas y privadas existentes en el Ecuador.

Se observa como el eje del sistema financiero es el Banco central por ser quien regula al resto de organización, además, dentro de esta estructuración también se toman en cuenta las 
instituciones de servicios auxiliares como los Transporte de especies monetarias y de valores, Servicios de cobranza, Cajeros automáticos, servicios contables y de Computación.

En la actualidad, a nivel local las instituciones financieras representan un total del $2,42 \%$ de las plazas de empleos que se generan en el país, siendo a proximadamente 73.406 personas las que intervienen o se sustentan de laborar en esta actividad económica.

\section{Créditos en instituciones financieras}

Los créditos se visualizan como la obtención de préstamos para que una empresa o persona pueda utilizar dichos recursos en actividades comerciales o de producción. Según Erazo (2019) "es una operación financiera que oferta una oportunidad de financiamiento al público e interesados por adquirir una determinada cantidad de dinero, a través del pago de una tasa de interés por el monto solicitado en un determinado tiempo", (pág. 11).

Conforme a lo que se expresa en el párrafo anterior, se entiende que los créditos de las instituciones financieras son acciones de costear las actividades del público objetivo, quienes cancelan el montón solicitado en un periodo de tiempo establecido por la entidad y con un interés determinado antes de su desembolso.

Los créditos dentro de las instituciones financieras varían según su tipo, para indagar sobre estos enfoques se presenta en la siguiente tabla la clasificación de esta variable.

Tabla 2.

Clasificación de créditos

\begin{tabular}{llll}
\hline Según el plazo de pago & Según el origen & Según su destino & Según la garantía \\
\hline - A corto plazo & $\bullet$ Bancario & $\bullet$ Producción & $\bullet$ Real o prendario \\
- Mediano plazo & $\bullet$ Comercial & $\bullet$ Consumo & $\bullet$ Hipoteca \\
- Largo plazo & & $\bullet$ Hipotecario & \\
& & $\bullet$ Automotriz & \\
& $\bullet$ Microy Pymes &
\end{tabular}

Nota: Adaptado de Erazo (2019) esta tabla presenta los tipos de créditos existentes

Se denota de la tabla anterior, que la modalidad de créditos que se ofrecen tanto para personas naturales y empresas, varía conforme a la finalidad que tenga su ejecución. Otra clasificación para los tipos de créditos es la que presenta Yance y Calle (2017), la cual, se presenta a continuación:

- Crédito Productivo. - La finalidad de esta modalidad es que las personas o empresas tengan la capacidad de cubrir en parte la deuda u obligaciones a contraer por efectuar una actividad económica.

- Crédito Comercial Ordinario. -Su acceso es para personas naturales que tengan la obligación de efectuar contabilidad e incluso a empresas que tengan dentro de sus ventas registros que excedan los $\$ 100$ mil dólares, además, su finalidad es adquirir activos como vehículos livianos ya sea utilizado para fines personales o comerciales.

- Crédito Comercial Prioritario. - Según Yance y Calle (2017) indican que:

Es el otorgado a personas naturales obligadas a llevar contabilidad o a personas jurídicas que registren ventas anuales superiores a USD 100,000.00 destinado a la 
adquisición de bienes y servicios para actividades productivas y comerciales, que no estén categorizados en el segmento comercial ordinario. (pág. 23)

- Crédito de Consumo Prioritario. - Se brinda a personas naturales, consignado a la adquisición de bienes, servicios o gastos no relacionados con una diligencia productiva 0 comercial y consumos no comprendidos en el segmento de consumo ordinario, adjuntos los créditos prendarios de joyas.

- Crédito Educativo. - Se ofrece a personas naturales para que ejecuten su formación y capacitación (puede ser profesional o técnica), además, se destina a empresas para el financiamiento que permita capacitar a su talento humano, estos aspectos deben ser apropiadamente célebre por los órganos competentes, así lo considera (Yance \& Calle, 2017).

- Crédito de Vivienda de Interés Público. - Se brinda mediante una garantía hipotecaria a personas naturales para la compra o edificación de estructuras o viviendas y destinadas a un primer uso.

- Crédito Inmobiliario. - Se ofrece al considerar una garantía hipotecaria por parte de personas naturales que quieran adquirir bienes inmuebles, con la finalidad de construir vivienda, siendo no categorizados en el fragmento de crédito Vivienda de Interés público (Yance \& Calle, 2017).

- Microcrédito.- Es el otorgado a una persona natural o jurídica con un nivel de ventas anuales inferior o igual a USD 100,000.00, o a un grupo de prestatarios con garantía solidaria. (Cooperativa de Ahorro y Crédito Lucha Campesina Ltda, 2015).

-

Fijación de tasas en los últimos periodos

Tabla 3.

Evolución de las tasasfijas del Ecuador

\begin{tabular}{lllllll}
\hline & \multicolumn{2}{l}{ Productivo Corporativo } & \multicolumn{2}{l}{ Productivo Empresarial } & \multicolumn{2}{l}{ Productivo PYMES } \\
\hline Año & $\begin{array}{l}\text { Tasas } \\
\text { referenciales }\end{array}$ & $\begin{array}{l}\text { Tasas } \\
\text { máximas }\end{array}$ & $\begin{array}{l}\text { Tasas } \\
\text { referenciales }\end{array}$ & $\begin{array}{l}\text { Tasas } \\
\text { máximas }\end{array}$ & $\begin{array}{l}\text { Tasas } \\
\text { referenciales }\end{array}$ & $\begin{array}{l}\text { Tasas } \\
\text { máximas }\end{array}$ \\
\hline $\mathbf{2 0 1 5}$ & $9,20 \%$ & $9,33 \%$ & $9,76 \%$ & $10,21 \%$ & $10,28 \%$ & $11,83 \%$ \\
\hline $\mathbf{2 0 1 6}$ & $8,48 \%$ & $9,33 \%$ & $9,84 \%$ & $10,21 \%$ & $11,15 \%$ & $11,83 \%$ \\
\hline $\mathbf{2 0 1 7}$ & $8,68 \%$ & $9,33 \%$ & $9,54 \%$ & $10,21 \%$ & $11,30 \%$ & $11,83 \%$ \\
\hline $\mathbf{2 0 1 8}$ & $8,81 \%$ & $9,33 \%$ & $9,85 \%$ & $10,21 \%$ & $11,23 \%$ & $11,83 \%$ \\
\hline $\mathbf{2 0 1 9}$ & $9.04 \%$ & $9,33 \%$ & $8,96 \%$ & $10,21 \%$ & $11,37 \%$ & $11,83 \%$ \\
\hline
\end{tabular}

Nota: Adaptado del Banco Central del Ecuador 2020

La tabla anterior permite observar una evolución de las tasas activas del Ecuador, las cuales, muestran una constancia en el máximo de interés que se pueden cobrar, la variación solo se evidencia en las tasas referenciales para las instituciones financieras estas se incrementan en cada periodo.

\section{Políticas implementadas en el último quinquenio para el sector financiero}

Para motivar a un mejor desarrollo de los diferentes sectores, las autoridades gubernamentales suelen plantear resoluciones y dictámenes enfocadas a las industrias que lo 
necesitan, por ello, en este apartado se presente examinar las directrices formuladas en los últimos cinco años para verificar a que sector se enfocaron y en qué consistían las medidas planteadas, estas se mencionan a continuación:

- Resolución No. 133-2015-M

- Plan de Reconstrucción y Reactivación Productiva post terremoto

- DECRETO 1070-2020

\section{Provincia del cañar}

La provincia del cañar, según informa el Instituto Ecuatoriano de Estadísticas y Censos INEC (2019) cuenta con un promedio de empresa equivalente a 616 entidades por cada 10 mil habitantes en su territorio, lo que refleja una amplia conformación empresarial a nivel local, ubicándose entre las 10 primeras provincias.

Figura 1

Porcentaje de empresas por cada 10 mil habitantes según provincias

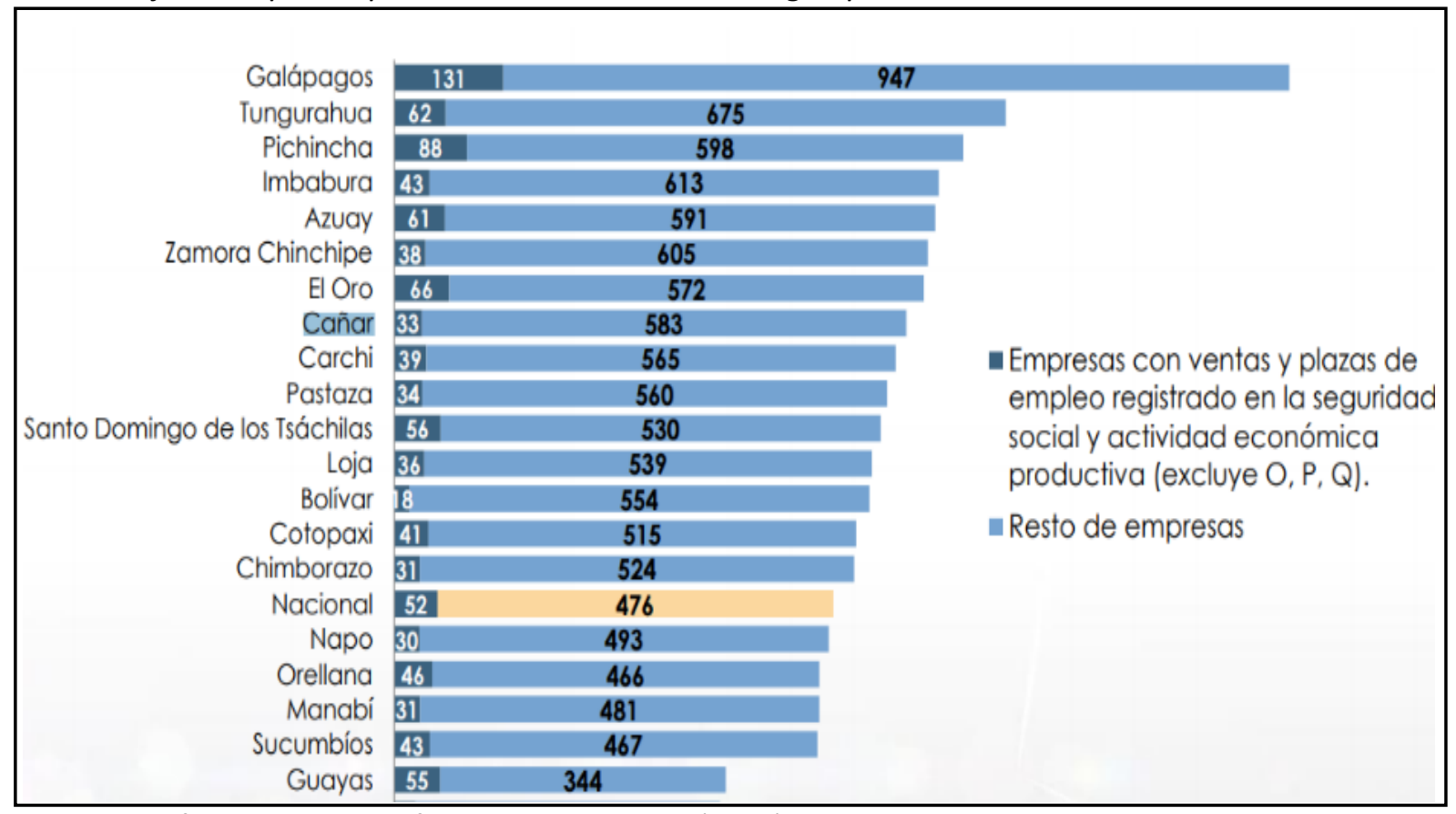

Nota: La figura presentada fue obtenida del INEC (2019)

Por otro lado, Pichincha, Guayas, Azuay, Manabí y El oro son las que concentran cerca del $90 \%$ de ventas que se generan en el contexto nacional. La entidad pública INEC también muestra datos sobre la actividad productiva generada conforme a cada una de las provincias del Ecuador, en este aspecto la localidad de Cañar tiene una representación de 0,80\% siendo Pichincha y Guayas con $30,72 \%$ y $26,54 \%$ las que mayor valor porcentual demuestran. La que menos aporte en acciones productivas genera es Bolívar con un 0,42\%. El 73,70\% de las empresas a nivel local provienen de las provincias de Pichincha, Guayas, Azuay, Manabí y el Oro, siendo las mismas que tienen una representación alta en ventas en el ámbito local. Esta información está disponible en la siguiente figura.

Figura 2. 


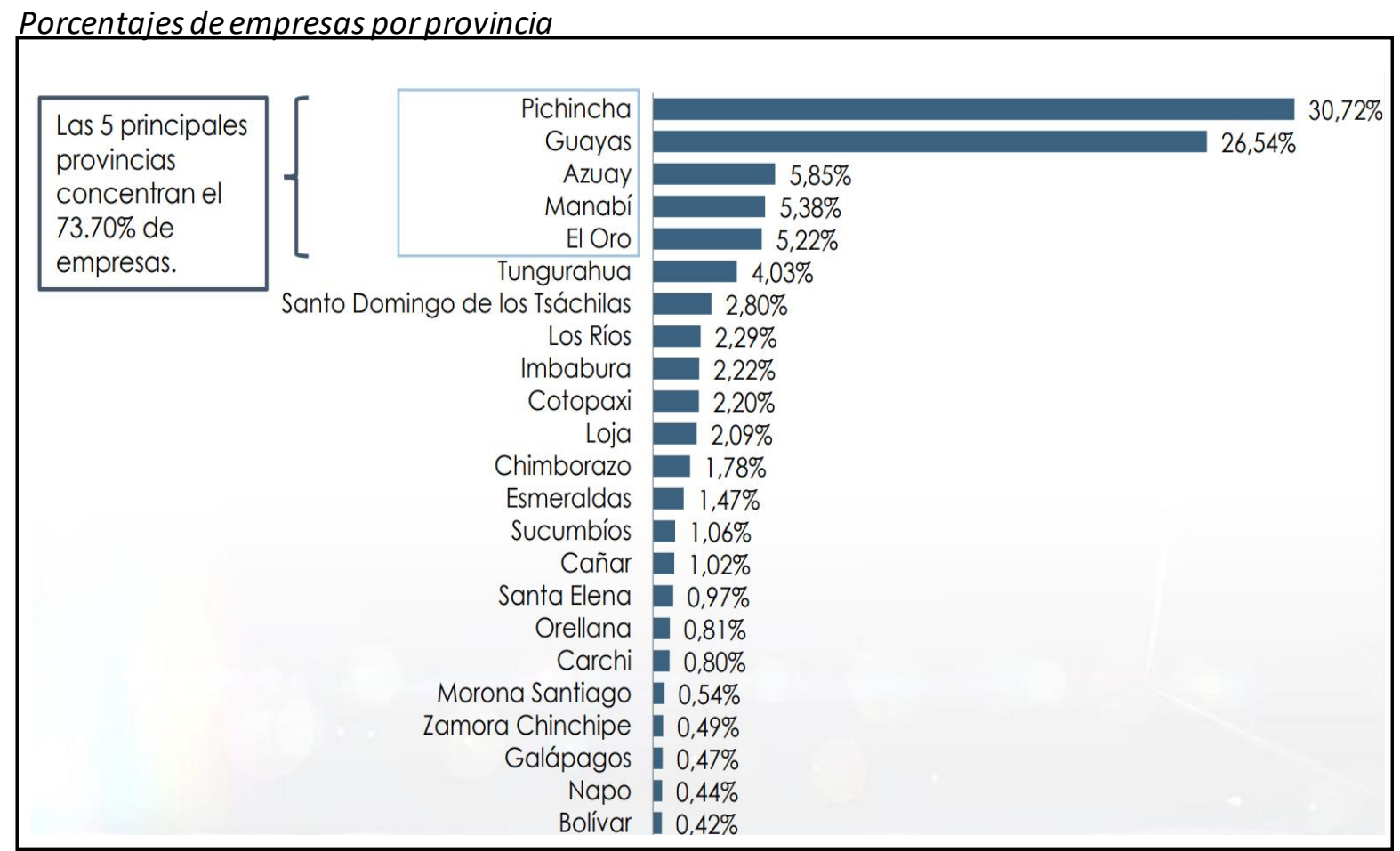

Nota: La información expuesta en la figura 2 fue adaptada del INEC (2019)

\section{Porcentaje de beneficio de los créditos de las empresas por provincia}

En Ecuador 44 de cada 100 ciudadanos tiene acceso directo a los servicios financieros de la banca ya sea esta pública o privada, así pues, se puede alardear que los otros 66 ciudadanos no tienen una cultura financiera propicia o sus medios no les permite acceder a los servicios ofrecidos por estas instituciones Para el año 2019 la banca pública otorgó 157.589 créditos de todo tipo es decir comerciales, ordinarios, microcréditos, educativos, productivos, etc.; mientras que la banca privada procesó 2.031 .3017 créditos a los ciudadanos. Sin embargo, como se puede observar en la siguiente figura existen provincias en las que el porcentaje de beneficio de créditos de la banca pública en mayor que el de la banca privada como por ejemplo en la provincia de Manabí, donde la banca privada alcanzo un beneficio del $16,43 \%$ mientras que la banca privada ejecutó el 7,24\%.

\section{Créditos productivos otorgados por provincias.}

La banca cumple un papel fundamental dentro de la economía de los países, pues sin estos muchos negocios nuevos o que deseen innovarse no podrían hacerlo por falta de recursos. Por esta razón a lo largo de los años en las bancas públicas y privadas en el Ecuador se han venido desarrollando políticas que les permitan a los emprendedores y dueños de negocios del sector productivo poder mejorar su actividad en diferentes ámbitos.

Para el año 2019 la tasa de morosidad fue de 3,1\% la misma que es considerada la más baja entre todos los países de la región, esto nos permite confirmar que de este total el promedio de morosidad por créditos productivos otorgados es del $1 \%$, que también es ubicado en uno de los más bajos de América Latina. Esta situación sumada a los incentivos entregados por la banca en el Ecuador para el sector productivo, ha hecho que la otorgación de este tipo de créditos haya aumentado en los últimos 2 años. Sin embargo, la banca privada 
sigue otorgando un nivel mayor de créditos que la banca pública, así como se puede observar en la siguiente figura.

\section{Figura 3.}

Número de créditos productivos otorgados por provincia

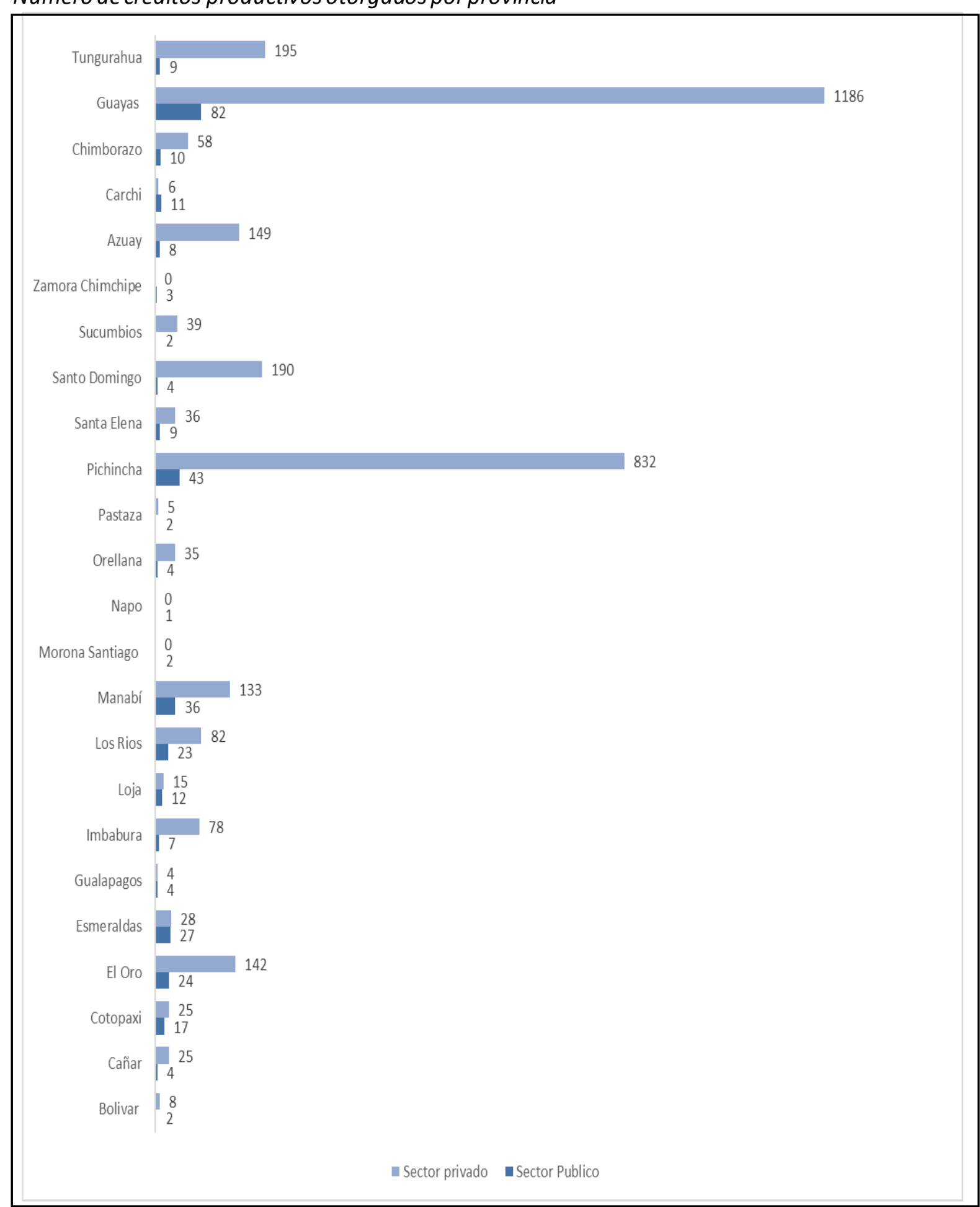

Nota: La información que se expone a continuación se obtuvo del portal de la Superintendencia de Bancos (2021) 


\section{Cantón La Troncal}

\section{Evolución de créditos públicos y privados en cantón La Trocal, provincia del Cañar}

La banca privada dentro de la provincia del Cañar ha mostrado un desenvolvimiento mayor que la publica en los últimos años, esto también es visible en el cantón La Troncal, donde se visualiza la siguiente información:

Las operaciones de crédito productivo dentro del cantón la Troncal muestran variaciones constantes en la banca pública, como se visualiza en la tabla anterior, este sector financiero tiene fluctuaciones constantes positivas y negativas, visualizando una reducción de 1 operación de crédito en el 2016 a 4 en el 2017. El sector privado muestra variaciones ascendentes y descendentes en estos periodos examinados, a partir el 2016, sin embargo, se visualiza una caída drástica de 9 operaciones crediticias en el 2018 a 3 para el 2019.

En una comparación entre ambos sectores, se visualiza que el privado tiene mayor flujo de operaciones, casi duplicando las transacciones de crédito de la banca pública, en el periodo de mayor afectación (2019), se denota que la banca privada fue fuertemente afectada (reducción en -6 operaciones), mientras que la banca pública creció del año 2018 de no tener créditos a tener 2 en el año 2019, por temas de crédito productivo. Para reflejar la evolución presentada en las tablas, se presenta la siguiente figura:

\section{Figura 4.}

Evolución de la banca en la Troncal

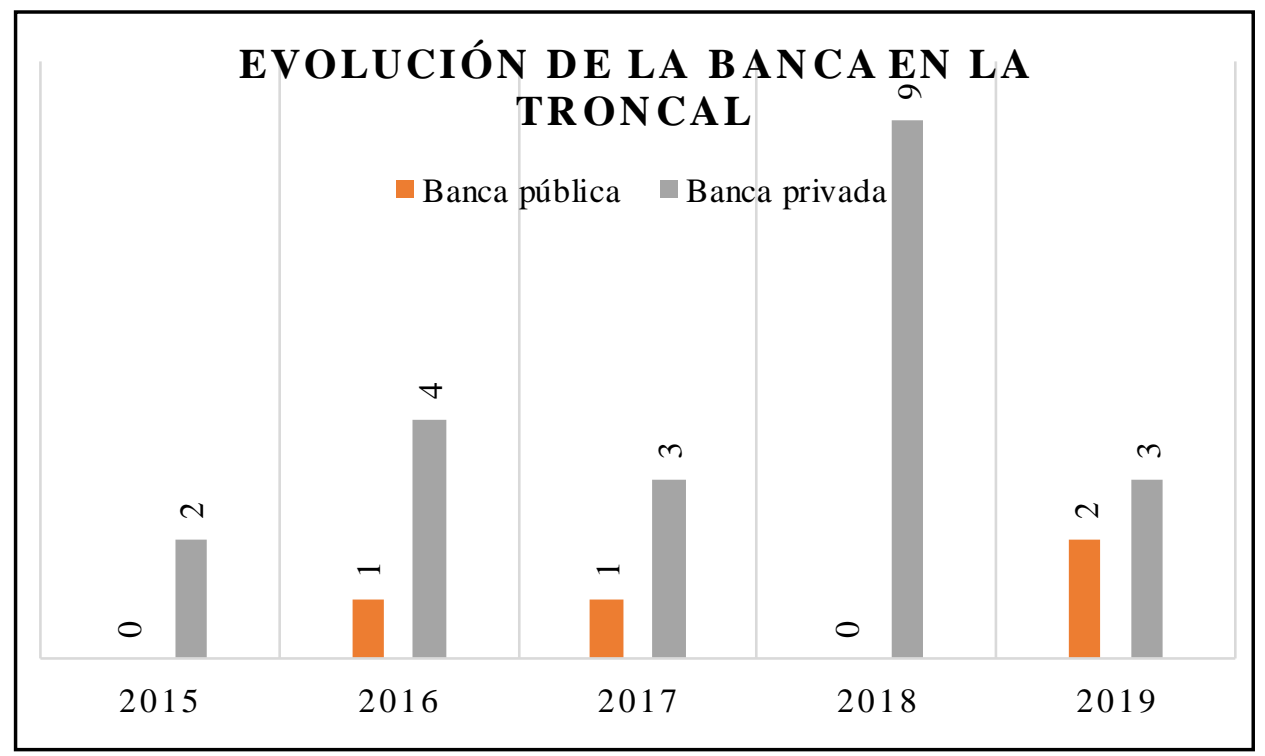

Nota: Esta información muestra el número de créditos otorgados por la banca en el cantón La Troncal fue adaptada de la Superintendencia de Bancos (2021)

\section{Fijación de tasas en los últimos periodos}

En La Troncal los créditos productivos en la banca privada aumentaron considerablemente en el año 2018, esto se da a que en este año la economía del país aumentó considerablemente comparada con la del año 2015. De esta manera se puede mencionar que las personas en La Troncal durante este año accedieron a 9 créditos productivos en la banca privada, que desde 
el año 2015 ha sido el mayor número de créditos productivos solicitados, pues a pesar que la banca pública ofrece mejores facilidades en el proceso de otorgación de créditos en cuestión de requisitos y formas de pago, los emprendedores escogen la banca privada por su baja tasa de interés.

En La Troncal en el año 2018 la banca pública no otorgo ningún crédito productivo, esta situación cambia en el año 2019 pues durante este año se otorgaron 2 créditos de este tipo, esta situación se presenta ya que la Corporación Financiera Nacional CFN, BanEcuador, el Banco de Desarrollo del Ecuador, la Corporación Nacional de Finanzas Populares (Conafips), el Banco del Instituto Ecuatoriano de Seguridad Social (Biess), el Banco del Pacífico y otras instituciones que forman el cuerpo de la banca pública del Ecuador destinaron un $14 \%$ más de recursos para la prestación de créditos productivos teniendo como enfoque principal beneficiar a los empresarios y comerciantes, permitiendo el fortalecimiento del sistema económico del cantón y del país.

Tabla 5.

Diferencias en de operaciones de crédito por sector en La Troncal

\begin{tabular}{cccc}
\hline Periodos & $\begin{array}{c}\text { Total de } \\
\text { operaciones }\end{array}$ & $\begin{array}{c}\text { Representación } \\
\text { porcentual banca } \\
\text { privada }\end{array}$ & $\begin{array}{c}\text { Representación } \\
\text { porcentual banca } \\
\text { pública }\end{array}$ \\
\hline $\mathbf{2 0 1 5}$ & 2.520 & $81 \%$ & $19 \%$ \\
\hline $\mathbf{2 0 1 6}$ & 1.503 & $63 \%$ & $37 \%$ \\
\hline $\mathbf{2 0 1 7}$ & 1.557 & $70 \%$ & $30 \%$ \\
\hline $\mathbf{2 0 1 8}$ & 1.587 & $71 \%$ & $29 \%$ \\
\hline $\mathbf{2 0 1 9}$ & 1.622 & $72 \%$ & $28 \%$ \\
\hline
\end{tabular}

Nota: Esta tabla muestra las operaciones de crédito en La Troncal des de el 2015 al 2019, adaptada del portal de la Superintendencia de Bancos (2021)

La tabla anterior permite observar que la banca privada muestra una mayor tendencia en la generación de operaciones de crédito productivo con respecto a la banca pública, denotándose diferencias que superan el 50\% en las transacciones realizadas para los últimos cinco años, es decir, que el rango de acción de las entidades gubernamentales no abastecen a la demanda de los emprendedores, que, no obstante de visualizar con mejores características a este tipo de instituciones, recurren a la de origen particular para poder solventar gestiones empresariales o productivas.

\section{METODOLOGÍA}

El presente de estudio exhibirá una metodología descriptiva de enfoque cuantitativo, al centrar el análisis en las entidades públicas y privadas que ofrecen los créditos para los prestatarios.

La población va hacer el número de entes productivos del sector primario localizados en La Troncal, para determinar su percepción sobre los créditos brindados por los sectores financieros, el número de establecimientos de acuerdo a los datos del Instituto Ecuatoriano de Estadísticas y Censos (INEC, 2019) se conforma de 289 entidades, que será la cantidad de elementos para determinar la muestra. Es decir, la muestra será de 68 emprendedores. Además, para ejecutar entrevistas se establece que solo se tomara en consideración a las entidades financieras de la provincia de Cañar, estimando como población un total de 4 instituciones ( 2 privadas y 2 públicas) a las que se le aplicara los formatos de entrevistas establecidos en el presente estudio. 
Como instrumento de investigación se estructura un cuestionario de encuestas a los entes productivos del sector primario localizados en La Troncal que permita determinar, conforme a la percepción de los emprendedores, que entidades financieras (según su sector) ofrecen mayores facilidades en tiempo de pago de créditos, tasas de interés, requerimientos y negociación de la deuda. Y para complementar la información sobre el fenómeno de estudio se establece un cuestionario de entrevistas dirigido a las entidades financieras del sector con la finalidad de obtener datos relacionados a las exigencias y lineamientos que siguen para otorgar créditos a las personas del sector productivo.

\section{RESULTADOS}

\section{Sector que tiene mayor exigente en requerimientos para créditos productivos}

Figura 5.

Sector que tiene mayor exigente en requerimiento para créditos productivos

\begin{tabular}{|rccc|}
\hline & \multicolumn{2}{c|}{ Sector que ofrece mayores requerimientos } \\
$100.00 \%$ & $60.29 \%$ & \\
$50.00 \%$ & $39.71 \%$ & & $0.00 \%$ \\
$0.00 \%$ & Banca pública & Banca privada & $\begin{array}{c}\text { Ninguno de los } \\
\text { anteriores }\end{array}$ \\
\cline { 2 - 4 } & & Porcentaje & \\
\hline
\end{tabular}

Los resultados de la encuesta permiten observar que el crédito que ofrecen las entidades presenta un mayor margen de requerimiento, Ilegando este al 60,29\% en LAS instituciones del sector privado. A reglón seguido está la banca pública con un 39,71\%, motivo, por consiguiente, la aprobación de préstamos por estos canales.

Figura 6.

Sector que ofrece mejor tasa de interés para créditos productivos

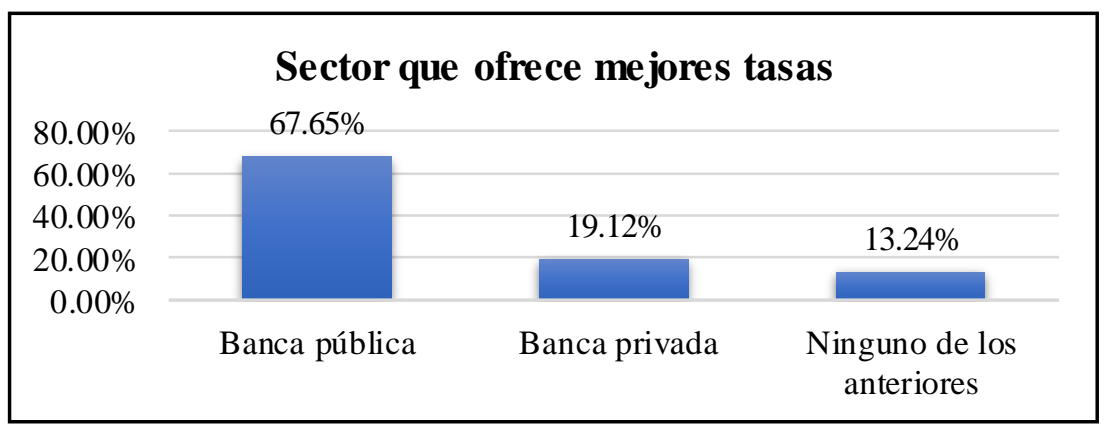

De acuerdo con los encuestados, el sector público ofrece mejores tasas de interés con respecto al ofrecimiento de crédito productivo representado en los resultados con un $67,65 \%$, mientras que la banca privada obtuvo un $19,12 \%$, por ende, se permite evidenciar una relación con la interrogante anterior que mencionaba que estas instituciones ofrecían mejores oportunidades. El 13,24\% no evidencia diferencia entre las tasas de ambos sectores. 
Figura 7.

Sector que permite negociación de la tasa de interés

\begin{tabular}{|c|c|c|c|}
\hline \multicolumn{4}{|c|}{ Sector con apertura a negociar tasa } \\
\hline \multirow{5}{*}{$\begin{array}{r}60.00 \% \\
40.00 \% \\
20.00 \% \\
0.00 \%\end{array}$} & & & \\
\hline & $29.41 \%$ & & \\
\hline & & $16.18 \%$ & \\
\hline & Banca pública & Banca privada & $\begin{array}{c}\text { Ninguno de los } \\
\text { anteriores }\end{array}$ \\
\hline & & orcentaje & \\
\hline
\end{tabular}

Los resultados de las encuestas permiten observar que el $54,41 \%$ menciona que ninguno de los sectores brinda facilidades de negociación para la tasa de interés, lo que permite denotar que esta es fija de acuerdo a lo que establecen las directrices de la institución, ta mbién se observa que el 29,41\% indica que la banca pública mues tra medidas alineadas a lo expuesto en el enunciado. Los que seleccionaron a cualquiera de los dos sectores indicaron que en ciertos casos le ofrecían la posibilidad de tasas reajustables. El 16,18\% es el menor porcentaje que se visualiza e indica la poca flexibilidad del sector privado.

Figura 8.

Sector que ofrece mayor facilidad de pago

\begin{tabular}{|cccc|}
\hline \multicolumn{3}{|c|}{$\begin{array}{c}\text { Sector con mayor felixibilidad en periodos de } \\
\text { pago }\end{array}$} \\
$100.00 \%$ & $69.12 \%$ & $22.06 \%$ & $8.82 \%$ \\
$0.00 \%$ & Banca pública & Banca privada & Ninguno de los anteriores \\
& & Porcentaje \\
\hline
\end{tabular}

El $69,12 \%$ de los encuestados menciona quela banca pública brinda mayores facilidades en el establecimiento de plazos para créditos, marcando un distanciamiento importante con respecto al $22,06 \%$ que menciona que es el sector privado brinda flexibilidades en este aspecto. Por ende, es otra cualidad en el que los solicitantes de créditos visualizan una mejor prestación por parte de instituciones públicas. También se observa que el $8,82 \%$ que no existe apertura por parte de ninguno de los sectores.

Figura 9.

Sector que ofrece mayor facilidad para negociar deuda por mora

\begin{tabular}{|c|c|c|c|}
\hline \multirow{4}{*}{$\begin{array}{r}100.00 \% \\
50.00 \% \\
0.00 \%\end{array}$} & \multicolumn{3}{|c|}{$\begin{array}{c}\text { Sector que muestra mayor predisposicion a } \\
\text { negociar }\end{array}$} \\
\hline & $51.47 \%$ & $26.47 \%$ & $22.06 \%$ \\
\hline & Banca pública & Banca privada & $\begin{array}{c}\text { Ninguno de los } \\
\text { anteriores }\end{array}$ \\
\hline & & orcentaje & \\
\hline
\end{tabular}


Los resultados de las encuestas permiten observar que el 51,47\% menciona que la renegociación por efectos de mora es más común en instituciones del ámbito público, siendo este otro de los factores en los que se observa un mejor servicio por parte de estas entidades. Este aspecto es fundamental considerando la realidad por la que pasan las empresas en la actualidad en el que requieren una mayor flexibilidad en sus prestaciones. Los resultados de las instituciones privadas fueron bajo con el $26,47 \%$, cercano al $22,06 \%$ que prefirió no seleccionar a ninguno de los sectores.

Figura 10.

Sector que ofrece mayor información sobre crédito

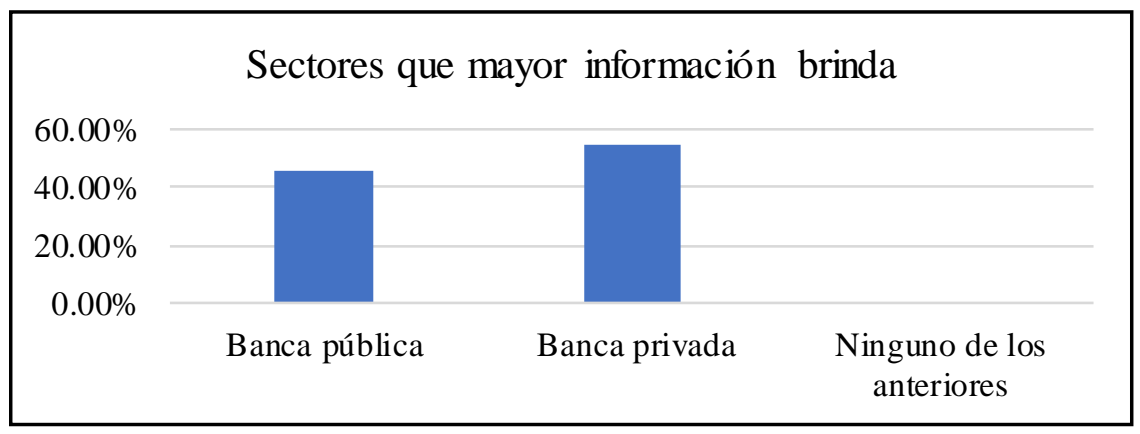

Sobre la accesibilidad a informa ción sobre los procesos y requerimi entos crediticos se considera por parte de los encuestados que en este aspecto el sector privado ofrece un mejor servicio, evidenciado con el 54,41\% en los resultados, siendo un aspecto res altable dentro de estas instituciones. Se debe mencionar que al hablar de otorgamiento de información se hace referencia a todos los canales o medios que se utiliza para mantener informado al cliente. Además, en esta característica la banca pública $(45,59 \%)$ debería ofrecer mejores canales que permitan el informarsea los usuarios.

Figura 11.

Sector que ofrece mayores incentivos decrédito productivo.

\begin{tabular}{|c|c|c|c|}
\hline \multicolumn{4}{|c|}{$\begin{array}{l}\text { Sectores con mayores incentivos en creditos } \\
69.12 \%\end{array}$} \\
\hline \multirow{4}{*}{$\begin{array}{r}60.00 \% \\
40.00 \% \\
20.00 \% \\
0.00 \%\end{array}$} & & & \\
\hline & $\theta$ & $16.18 \%$ & \\
\hline & & & $0.00 \%$ \\
\hline & Banca pública & Banca privada & $\begin{array}{c}\text { Ninguno de los } \\
\text { anteriores }\end{array}$ \\
\hline
\end{tabular}

El 69,12\% menciona que el sector público genera mejores incentivos para créditos productivos, lo cual, se puede vincular al hecho de ser una empresa pública que se adaptará a las medidas que facilitará el gobierno local para los diferentes sectores económicos, en cambio, el sector privado lo implementa conforme a sus beneficios financieros. Por ende, se comprende el bajo promedio que obtuvo dentro de los resultados con el $16,18 \%$.

Figura 12.

Sector que ofrece mayor facilidad de pago

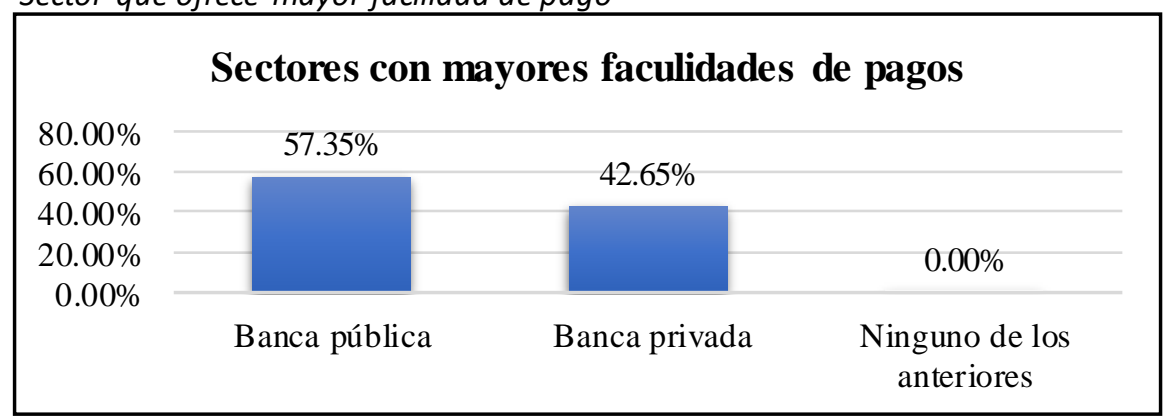


De acuerdo con el 57,35\%, el sector que mayor facilidad de pago ofrece es el sector público que superan el $42,65 \%$ que selecciono al privado, por ende, las instituciones de estas características ofrecen mejores servicios a los clientes de la localidad, marcando diversas diferencias en diferentes cuestiones de crédito.

\section{Resultados y análisis de entrevista a sector privado y público}

Tabla 6.

Análisis de entrevista a sector privado y público

\begin{tabular}{|c|c|c|}
\hline Ítems & Instituciones publicas & Instituciones privadas \\
\hline $\begin{array}{l}\text { Tasa de interés para créditos } \\
\text { productivos }\end{array}$ & $11,23 \%$ & $9,76 \%$ \\
\hline Plazos que otorga & $\begin{array}{l}\text { Capital de trabajo tiene un máximo } \\
\text { de } 18 \text { meses o añoymedio. } \\
\text { Para activos como equipos y } \\
\text { mobiliarios hasta } 36 \text { meses o tres } \\
\text { años. } \\
\text { Para inmobiliarios, no puede exceder } \\
\text { los } 60 \text { meseso cinco años. } \\
\text { Solo en caso de local comercial se } \\
\text { estipula hasta } 10 \text { años. }\end{array}$ & $\begin{array}{l}\text { Ca pital de trabajo hasta } 3 \text { a ños } \\
\text { Adquisición de a ctivos hasta } 10 \\
\text { a ños. } \\
\text { Pa ra proyectos en general, de } 3 \text { a } \\
10 \text { años. }\end{array}$ \\
\hline Facilidades de pagos & $\begin{array}{l}\text { De acuerdo al tarifario que } \\
\text { estructura la institución, se platean } \\
\text { cuotas de cobro mensuales. }\end{array}$ & $\begin{array}{l}\text { Se adaptan al flujo de efectivo, } \\
\text { puede ser mensuales, } \\
\text { bimensuales, trimestrales, } \\
\text { semestraleso a nuales. }\end{array}$ \\
\hline Requisitos & $\begin{array}{l}\text { Estipula cerca de diez requisitos que } \\
\text { debe cumplir el solicitante entre los } \\
\text { que están los certificados de } \\
\text { Superintendencia de Compañías, } \\
\text { IESS y SRI, además, del garante o } \\
\text { avaluó de activos. }\end{array}$ & $\begin{array}{l}\text { Es tipula ce rca de cinco requisitos } \\
\text { centrados en información del } \\
\text { solicitante ygarante, además del } \\
\text { plande factibilidad. }\end{array}$ \\
\hline $\begin{array}{l}\text { Tiempo de respuesta de la } \\
\text { solicitud de crédito }\end{array}$ & De 2 a 10 días laborables. & De 2 a 5 días laborables. \\
\hline
\end{tabular}

\section{DISCUSIÓN}

La información examinada permite observar que las principales provincias a nivel local en las que se promueven estas instituciones se centran en los mercado de Pichincha y Guayas que son los sectores mayormente comerciales a nivel local, por lo que, contar con servicios financieros cercanos y variados permitirán impulsar sus actividades económicas, también se visualiza los tipos de créditos existentes, en los que se menciona créditos informales como parte de recursos que se ofrecen en los mercados, siendo este peligroso para el usuario al no tener fundamento documental, no obstante, ante posibles excesos de trámites y requisitos, es una opción a la que suelen recurrir los habitantes.

Entre los términos abarcados se exponen las definiciones que permitan diferenciar entre lo que comprende institución financiera y sistema financiero, entendiéndose que la última menciona abarca a la primera al ser una red que gestiona las diferentes entidades del sector y los marcos legales que se exponen para regular las operaciones que ejercen.

El marco permite concluir que las leyes fijan puntos máximos de referencias que no deben ser sobrepasado por parte de las instituciones que incurren en brindar servicios en el contexto nacional, por ende, si existe regulaciones en este sentido, no obstante, solo se 
estipula el tope de las tasas y no obliga a la estipulación de porcentajes iguales por parte de las entidades.

El conocimiento del marco legal permitirá que las personas conozcan cómo se regula la estructuración, gestión, transacción, funcionamiento y extinción de las entidades financieras. Entre los aspectos relevantes tratado se exhibe como gubernamental, por parte de las autoridades, está penado la utilización de tasas diferentes a las que se pactan por el servicio, inclusive, sancionan la no divulgación de información sobre las tarifas de los servicios crediticios, por lo que, se entiende que da protección a los usuarios o ciudadanos.

\section{CONCLUSIONES}

- La investigación permitió observar que características distinguen a las empresas o instituciones financieras privadas y públicas, denotándose que las privadas buscan la generación de créditos para la entidad mientras que la de origen público tienen la finalidad de brindar asistencia en operaciones financieras a los ciudadanos. En lo que respecta a créditos productivos se visualiza que las tasas de interés de las entidades particulares son superiores promediándose en un $9 \%$ mientras que las públicas ofrecen un $2 \%$ mayor en estas operaciones.

- Se pudo observar las presunciones que se relacionan con la generación de créditos, entre las que resalta la Teoría del Racionamiento del Crédito como una medida que restringe el crédito para lograr un equilibrio entre la oferta y demanda de estos servicios. Por otro lado, los componentes o requisitos dentro de las solicitudes de crédito, para las entidades privadas se exhibe una exigencia mayor al requerir certificados provenientes de instituciones gubernamentales como la Superintendencia de compañías, IESS y SRI, siendo indispensable para poder acceder al préstamo. Por su parte, el sector público muestra requerimientos menos rigurosos al solo solicitar documentación del solicitante y del garante, además del flujo del proyecto, estos aspectos también forman parte de los requisitos del primer sector mencionado. En lo que respecta al tiempo de aceptación del crédito, se menciona promedios similares siendo lo mínimo dos días.

- Se concluye que los plazos de las instituciones públicas avizoran mayor flexibilidad, al proponer un mínimo de 3 años hasta un máximo de 10 años, en el sector privado estos varían de un mes a 18 meses hasta cinco años, solo en caso de locales comerciales admiten periodos de 10 años. En lo referente a las tasas estas son de $11,23 \%$ en los bancos privados y de $9,25 \%$ en los públicos, es decir, se ofrecen mejores características las instituciones del gobierno. Así pues, las leyes implementadas en el último quinquenio, visualizan poco direccionamiento en convenios con el sector privado, destacando el programa Reactívate Ecuador impulsado junto al banco del pacifico en el 2020 que otorgará \$1.500 para incentivar el sector productivo.

\section{REFERENCIAS}

Aguilera, F. (2015). El impacto de la crisis financiera y económica internacional en la banca del Ecuador. Quito: Editorial de la Universidad Andina Simón Bolívar, Sede Ecuador. 
Banco Mundial. (2019). Tasa de alfabetización, total de adultos (\% de personas de 15 años o más). Obtenido de https://datos.bancomundial.org/indicador/SE.ADT.LITR.ZS

Carillo, V., Mancero, H., \& Mancero, D. (2019). Análisis de la crisis bancaria privada ecuatoriana (1994-2000) y sus efectos socioeconómicos. Revista Cofin Habana, Vol. 13, 1-13. Obtenido de http://scielo.sld.cu/scielo.php?script=sci_arttext\&pid=S207360612019000300017

Collaguazo, A. (2017). Prestamos financieros como opción para enfrentar la crisis económica del año 2016, en los distribuidores de insumos médicos (Pymes) en el DM.Q. (Tesis de pregrado). Obtenido de Universidad Politécnica Salesiana: https://dspace.ups.edu.ec/bitstream/123456789/13657/1/UPS-QT11478. pdf

Cooperativa de Ahorro y Crédito Lucha Campesina Ltda. (2015). Segmentación de la Cartera de Crédito. La Troncal, Cañar, Ecuador. Obtenido de http://www.coacluchacampesina.fin.ec/

Cunalata, P., \& Quijije, S. (2016). Factibilidad para la creación de Cooperativa de Ahorro y Crédito. Sector Artesanal (Tesis de pregrado). Obtenido de Universidad de Guayaquil: http://repositorio.ug.edu.ec/bits trea m/redug/14091/1/TESIS\%20Cpa\%20117\%20\%20Factibilidad\%20para\%20la\%20creaci\%C3\%B3n\%20de\%20Cooperativa\%20de\%20 Ahorro\%20y\%20Cr\%C3\%A9dito.\%20Sector\%20Artesanal.pdf

ENEMDU . (2020). Encuesta Nacional de Empleo, Desempleo y Subempleo. Obtenido de INEC: https://www.ecuadorencifras.gob.ec/documentos/webinec/POBREZA/2020/Diciembre-2020/202012_PobrezayDesigualdad.pdf

Erazo, P. (2019). Análisis de la Evolución del Crédito para Financiar Actividades Productivas en Ecuador en el Periodo 2014 - 2018 (Tesis de pregrado). Obtenido de Universidad Católica de Santiago de Guayaquil: http://repositorio.ucsg.edu.ec/bitstream/3317/13287/1/T-UCSG-PRE-ESP-CFI529.pdf

Guilcaso, J., Ronquillo, J., \& Montaluisa, R. (2018). Crisis económica política y social del Ecuador periodo 1998 hasta parte del primer semestre del 2018. Revista científica de investigación actualización del mundo de las ciencias Vol 3, 517-569. doi:http://reciamuc.com/index.php/RECIAMUC/article/view/247

INEC. (2011). Encuesta de Victimización y Percepción de Inseguridad. Obtenido de Instituto Nacional de Estadística y Censos: https://anda.inec.gob.ec/anda/index.php/catalog/673/download/12133

INEC. (2019). Diretorio de empresas y establecimientos. Obtenido de Instituto Ecuatoriano de Estadisticas y Censos: https://www.ecuadorencifras.gob.ec/documentos/webinec/Estadisticas_Economicas/DirectorioEmpresas/Directorio_Empresas_2018/Princi pales_Resultados_DIEE_2018.pdf

INEC. (2021). Estadísticas de Seguridad Integral - Denuncias de delitos de mayor incidencia. Obtenido de Instituto Nacional de Estadísticas y Censos: https://www.ecuadorencifras.gob.ec/justicia-y-crimen/

López, J., \& Farías, E. (2018). Análisis de alternativas de financiamiento para las Pymes del sector Comercial e Industrial de Guayaquil (Tesis de pregrado). Obtenido de Universidad de Guayaquil: http://repositorio.ug.edu.ec/bitstrea m/redug/30172/1/TesisAlternativas\%20de\%20Financiamiento\%20para\%20PYMES.pdf

Lovato, G. (2015). Diagnóstico del sistema financiero ecuatoriano su responsabilidad social e inadecuada aplicación de procesos administrativos a través de expedicionesy reformas 
legales. (Tesis de pregrado). Obtenido de Universidad Central del Ecuador: http://www.dspace.uce.edu.ec/bitstream/25000/5569/1/T-UCE-0013-Ab-397.pdf

Marichal, C., \& Gambi, T. (2017). Historia bancaria y monetaria de América Latina (siglos XIX y XX): Nuevas Perspectivas. España: Editorial de la Universidad de Cantabria. Obtenido de https://www.editorial.unican.es/libro/historia-bancaria-y-monetaria-de-americalatina-siglos-xix-y-xx-nuevas-perspectivas-0

Ortega, C. (2015). El Sistema Financiero Ecuatoriano, La Superintendencia De Bancos Y Banco Central Del Ecuador, En El Período 2007-2012 (Maestria). Obtenido de Universidad de Guayaquil:

http://repositorio.ug.edu.ec/bits trea m/redug/7905/1/Tesis\%20Christian\%200rtega\% 20Final.pdf

PDOT La Troncal. (2014). Plan De Desarrollo Y Ordenamiento Territorial Del Cantón La Troncal 2014- 2019. Obtenido de Gobierno Autónomo Descentralizado de La Troncal: https://multimedia.planificacion.gob.ec/PDOT/descargas.html

Posso, R. (2016). Historia De La Creación De La Banca Central Latinoamericana -El pretérito es la base de un presente prominente. Revista de la Facultad de Ciencias Económicas y Administrativas. Universidad de Nariño., XVII(2), 166-187. Obtenido de http://www.scielo.org.co/scielo.php?script=sci_arttext\&pid=S012486932016000200009

Reinoso, J. (2018). Análisis de la incidencia de las tasas de interés y del volumen de crédito en el crecimiento económico de los sectores productivos del Ecuador periodo 2002-2015 (Tesis de posgrado). Obtenido de Universidad Católica de Santiago de Guayaquil: http://repositorio.ucsg.edu.ec/bitstream/3317/11982/1/T-UCSG-POS-MFEE-153.pdf

Superintendencia de Bancos. (2021). Portal estadístico. Obtenido de Reportes de Volumen de Crédito:

https://estadisticas.superbancos.gob.ec/portalestadistico/portalestudios/?page_id=3 27

Tello, J., Hernani, M., \& Limaco, B. (2017). Capacidad Transaccional: Evidencias Del Sistema Financiero Peruano. RAE-Revista de Administração de Empresas., Vol. 1, 37-50. Obtenido de http://www.scielo.br/j/rae/a/k9yfLS9RpmnvNKzWcf67tvK/?lang=es

Yance, K., \& Calle, M. (2017). Análisis para identificar las variables que inciden en el índice de cartera vencida de la cooperativa de ahorro y crédito "Lucha campesina LTDA" Agencia La Troncal (Tesis de pregrado). Obtenido de Universidad de Guayaquil: http://repositorio.ug.edu.ec/bitstrea m/redug/17707/1/\%E2\%80\%9CAn\%C3\%A1lisis\% 20para\%20identificar\%20las\%20variables\%20que\%20inciden\%20en\%20el\%20\%C3\%A Dndice\%20de\%20cartera\%20vencida\%20de\%20la\%20cooper.pdf 